TRANSACTIONS OF THE

AMERICAN MATHEMATICAL SOCIETY

Volume 360, Number 10, October 2008, Pages 5477-5488

S 0002-9947(08)04548-0

Article electronically published on April 9, 2008

\title{
MIXED NORM ESTIMATES FOR CERTAIN GENERALIZED RADON TRANSFORMS
}

\author{
MICHAEL CHRIST AND M. BURAK ERDOĞAN
}

\begin{abstract}
We obtain essentially optimal local mixed norm inequalities for certain generalized Radon transforms defined by integration over curves.
\end{abstract}

\section{INTRODUCTION}

In this paper we investigate the mapping properties in Lebesgue-type spaces of certain generalized Radon transforms defined by integration over curves.

Let $X$ and $Y$ be open subsets of $\mathbf{R}^{d}, d \geq 2$, and let $Z$ be a smooth submanifold of $X \times Y \subset \mathbf{R}^{2 d}$ of dimension $d+1$. Assume that the projections $\pi_{1}: Z \rightarrow X$ and $\pi_{2}: Z \rightarrow Y$ are submersions at each point of $Z$. For each $y \in Y$, let

$$
\gamma_{y}=\{x \in X:(x, y) \in Z\}=\pi_{1} \pi_{2}^{-1}(y) .
$$

In this case, $\gamma_{y}$ are smooth curves in $X$ which vary smoothly with $y \in Y$. For every $y \in Y$, choose a smooth, nonnegative measure $\sigma_{y}$ on $\gamma_{y}$ which varies smoothly with $y$ in the natural sense. A generalized Radon transform $T$ (see, e.g., 2, 10, 15]) is defined as an operator taking functions on $X$ to functions on $Y$ via

$$
T f(y)=\int_{\gamma_{y}} f d \sigma_{y} .
$$

The adjoint of this operator has a similar form:

$$
T^{*} g(x)=\int_{\gamma_{x}^{*}} g d \sigma_{x}^{*}
$$

where

$$
\gamma_{x}^{*}=\{y:(x, y) \in Z\}=\pi_{2} \pi_{1}^{-1}(\{x\}) \subset Y
$$

and $\sigma_{x}^{*}$ is a nonnegative measure on $\gamma_{x}^{*}$ with a smooth density which varies smoothly with $x$.

Tao and Wright [16] have formulated and proved a nearly optimal characterization of the local $\left(L^{p}, L^{q}\right)$ mapping properties of these operators. We extend their result to the mixed-norm setting and obtain essentially optimal local mixednorm inequalities for these operators, under one additional dimensional restriction. Previously this result was obtained for a model operator in [17, 6, 4. See [1, 4, 6, 12, 13, 14, 16, 17, for various examples and prior work, and 2] for a partially alternative development of the unmixed norm theory.

Received by the editors September 7, 2005 and, in revised form, November 9, 2006.

2000 Mathematics Subject Classification. Primary 44A12; Secondary 47G10.

The first and second authors were partially supported by NSF grants DMS-040126 and DMS0540084, respectively.

(C)2008 American Mathematical Society Reverts to public domain 28 years from publication 
Mixed norms on $Y$. Throughout the entire discussion, $X, Y$ will denote sufficiently small neighborhoods of $x_{0}, y_{0}$ for some fixed point $\left(x_{0}, y_{0}\right) \in Z$. Let $\Pi: Y \rightarrow \mathbf{R}$ be a submersion such that the fibers $\Pi^{-1}(t)$ are transverse to the curves $\gamma_{x}^{*}$. This means that the restriction of $\Pi$ to $\gamma_{x}^{*}$ is a diffeomorphism for each $x \in X$. Choose coordinates so that $\Pi\left(y_{0}\right)=0$. Let $\lambda_{t}$ be Lebesgue measure on the $(d-1)$-dimensional surface $\Pi^{-1}(t)$. To a function $f: Y \mapsto \mathbf{R}$ we associate the mixed norms

$$
\|f\|_{L^{q} L^{r}(Y)}=\|f\|_{q, r}:=\left[\int_{\mathbf{R}}\left[\int_{\Pi^{-1}(t)}|f(s)|^{r} d \lambda_{t}(s)\right]^{q / r} d t\right]^{1 / q} .
$$

The integral with respect to $t$ is taken over a small neighborhood of the origin in $\mathbf{R}$; we may assume that this neighborhood is contained in $[-1,1]$. Here, and throughout this paper, $t$ is restricted to lie in a one-dimensional manifold. This is not a natural restriction, but our analysis yields reasonably satisfactory results only in this special case.

This situation is quite general, and is satisfied for generic choices of the jets, up to a certain order depending only on $d$, of $X, Y, \Pi$ at $\left(x_{0}, y_{0}\right)$. Begin with any manifold $Z$ of dimension $d+1$, and a point $z_{0} \in Z$. In a neighborhood of $z_{0}$ construct two real vector fields $V_{X}, V_{Y}$ which are linearly independent at $z_{0}$, and which generate a Lie algebra that spans the tangent space to $Z$ at $z_{0}$. Such vector fields exist in any dimension, and hence for any $d$ there exists $N$ such that any pair $\left(V_{X}, V_{Y}\right)$ with generic jet up to order $N$ at $z_{0}$ will satisfy this condition. Choose two codimensionone manifolds $X, Y \subset Z$ in a neighborhood of $z_{0}$, such that the integral curves of $V_{X}, V_{Y}$ passing through $z_{0}$ are transverse to $X, Y$, respectively, at $z_{0}$. There are natural submersions $\pi_{X}, \pi_{Y}$ from $Z$ to $X, Y$, defined by flowing from $z \in Z$ to $X, Y$ along the integral curves of $V_{X}, V_{Y}$, respectively. The only requirement on $\Pi: Y \rightarrow \mathbf{R}$ is then that $V_{X}\left(\pi_{Y} \circ \Pi\right)\left(z_{0}\right) \neq 0$. Equivalently, $D \Pi\left(z_{0}\right)$ is required not to annihilate the push-forward under $\pi_{Y}$ of $V_{X}\left(z_{0}\right)$. This is a single linear condition on $D \Pi\left(z_{0}\right)$, so any generic map $\Pi$ satisfies the hypothesis. In particular, one can consider such mixed norms for each of the concrete examples discussed in [16].

A fundamental example which motivates our investigation has $X=\mathbf{R}^{d}, Y$ equal to the manifold of all lines in $\mathbf{R}^{d}$, and $Z=\{(x, L) \in X \times Y: x \in L\}$. To any line $L$ is associated $\Pi(L)$, the unique line parallel to $L$ containing the origin. $\Pi$ maps $Y$ to the manifold $G$ of all one-dimensional subspaces of $\mathbf{R}^{d}$. The x-ray transform maps $f: \mathbf{R}^{d} \rightarrow \mathbf{C}$ to $T f: Y \rightarrow \mathbf{C}$, with $T f(L)$ equal to the integral of $f$ over $L . Y$ can naturally be parametrized as the set of all $(\theta, x) \in G \times \mathbf{R}^{d-1}$ such that $x \perp \theta$; $L=x+\theta$ is a line parallel to $\theta$. Thus one has mixed norms $L_{\theta}^{q} L_{x}^{r}$ for $Y$. Let $B$ be a ball of finite radius in $\mathbf{R}^{d}$. A strong form of conjectured bounds for the Kakeya maximal function would say that $T$ is bounded from $L^{q}(B)$ to $L_{\theta}^{q} L_{x}^{r}(Y)$, for all $r<\infty$ and $q>d$. The inequality holds for $d=2$ and is unknown for $d \geq 3$. This example fails to satisfy our hypotheses in two respects for $d \geq 3$. Firstly, $Y$ has dimension $2 d-2>d$. This means that the operator formally adjoint to $T$ involves integration over submanifolds of dimension $d-1>1$, rather than over curves. Secondly, $G$ has dimension strictly greater than one. In our view, this example amply motivates a general investigation of mixed-norm inequalities for generalized Radon transforms, even though such a general framework neglects special features of the motivating problem. 
We say that $T$ is of strong mixed type $(p, q, r)$ if $T$ maps $L^{p}(X)$ to $L^{q} L^{r}(Y)$ boundedly. We are mainly interested in local estimates. We assume throughout the discussion that $T$ is $L^{p}$-improving, which means that for each $p \in(1, \infty)$, there exists $q>p$ such that $T$ maps $L^{p}(X)$ to $L^{q}(Y)$. See [5] and [15] for characterizations of this property.

Our theorem is a characterization of the exponents $(p, q, r)$ for which $T$ is bounded. Before proceeding, let us record some simple facts about these exponents.

(i) Because of the transversality hypothesis described above, $T$ is of strong mixed type $(p, \infty, p)$ for all $p \in[1, \infty]$.

(ii) Since we are working in a bounded region, whenever $T$ is of strong mixed type $(p, q, r)$, it is also of strong mixed type $\left(p_{1}, q_{1}, r_{1}\right)$ whenever $p_{1} \geq p, q_{1} \leq q$, and $r_{1} \leq r$.

(iii) Because of (i) and (ii), $T$ is of strong mixed type $(p, q, r)$ whenever $p \geq r$.

Two-parameter Carnot-Carathéodory balls. Tao and Wright [16] related the set of all exponents $(p, q)$ for which the operator $T$ maps $L^{p}(X)$ to $L^{q}(Y)$ to the geometry of $Z$. To describe this relation, choose smooth nowhere-vanishing linearly independent real vector fields $V_{1}, V_{2}$ on $Z$ whose integral curves are the fibers of $\pi_{1}, \pi_{2}$ respectively. Equivalently, at each point $z \in Z, V_{j}$ spans the nullspace of $D \pi_{j}$, for $j=1,2$. The $L^{p}$-improving property is equivalent to $V_{1}, V_{2}$ satisfying the bracket condition, i.e., $V_{1}$ and $V_{2}$ together with their iterated commutators span the tangent space to $Z$ at each point in $Z$; see [5].

Definition 1. Let $z_{0} \in Z$ and $0<\delta_{1}, \delta_{2} \ll 1$. The two-parameter CarnotCarathéodory ball $B\left(z_{0}, \delta_{1}, \delta_{2}\right)$ consists of all the points $z \in Z$ such that there exists an absolutely continuous function $\varphi:[0,1] \rightarrow Z$ satisfying

(i) $\varphi(0)=z_{0}, \varphi(1)=z$

(ii) for almost every $t \in[0,1]$,

$$
\varphi^{\prime}(t)=a_{1}(t) V_{1}(\varphi(t))+a_{2}(t) V_{2}(\varphi(t))
$$

with $\left|a_{1}(t)\right|<\delta_{1},\left|a_{2}(t)\right|<\delta_{2}$.

The metric properties of Carnot-Carathéodory balls were studied extensively in 11. The discussion there is phrased in terms of the one-parameter family of balls naturally associated to a family of vector fields satisfying the bracket condition. These balls depend on a center point, a radius $r$, and a family $\left\{W_{j}\right\}$ of vector fields. They can equivalently be viewed as depending on a center point and the family $\left\{r W_{j}\right\}$ of vector fields, with the radius redefined to be identically one. In these terms, the proofs in 11] go through more generally, for balls of radius one assigned to families of vector fields $\left\{U_{j}^{\alpha}: 1 \leq j \leq J\right\}$ satisfying the bracket condition for each parameter $\alpha$, with appropriate uniformity as $\alpha$ varies. In particular, for the vector fields $\left\{\delta_{1} V_{1}, \delta_{2} V_{2}\right\}$, provided that $0<\delta_{1}, \delta_{2} \leq c_{0}$ for sufficiently small $c_{0}$, the conclusions of [1] hold uniformly in $\delta_{1}, \delta_{2}$ under a supplementary hypothesis of weak comparability, which is discussed below. See [16, 2].

It will be convenient in our proof to parametrize the curves $\gamma_{x}^{*}$ by $t$ so that $\Pi\left(\gamma_{x}^{*}(t)\right) \equiv t$. With this parametrization, the measure $\sigma_{x}$ on $\gamma_{x}^{*}$ is equivalent to $d t$, uniformly in $x$. We rescale $V_{1}$ if necessary so that for each $z \in Z$ and sufficiently small $s \in \mathbf{R}$,

$$
\Pi \pi_{2}\left(e^{s V_{1}} z\right)=\Pi \pi_{2}(z)+s
$$


Definition 2. Let $0<\theta \leq 1$, and let $A$ be a positive constant. We say that $0<\delta_{1}, \delta_{2} \ll 1$ are $(\theta, A)$-weakly comparable, and write $\delta_{1} \sim_{\theta, A} \delta_{2}$, if $\delta_{1} \leq A \delta_{2}^{\theta}$ and $\delta_{2} \leq A \delta_{1}^{\theta}$.

The following lemma collects basic facts about the balls $B\left(z, \delta_{1}, \delta_{2}\right)$.

Lemma 1. Let $K$ be a compact subset of $Z$. Assume that $\delta_{1} \sim_{(\theta, A)} \delta_{2}$ are sufficiently small, and let $z \in K$. Then $B=B\left(z, \delta_{1}, \delta_{2}\right)$ satisfies

(i) $|B| \sim\left|B\left(z, 2 \delta_{1}, 2 \delta_{2}\right)\right|$

(ii) $|B| \sim\left|\pi_{1}(B)\right| \delta_{1} \sim\left|\pi_{2}(B)\right| \delta_{2}$,

(iii) $\left|\Pi\left(\pi_{2}(B)\right)\right| \sim \delta_{1}$,

(iv) $\left\|\chi_{\pi_{2}(B)}\right\|_{q^{\prime}, r^{\prime}} \sim|B|^{1-\frac{1}{r}} \delta_{1}^{\frac{1}{r}-\frac{1}{q}} \delta_{2}^{\frac{1}{r}-1}$,

$$
\frac{|B|}{\left|\pi_{1}(B)\right|^{\frac{1}{p}}\left\|\chi_{\pi_{2}(B)}\right\|_{q^{\prime}, r^{\prime}}} \sim|B|^{\frac{1}{r}-\frac{1}{p}} \delta_{1}^{\frac{1}{p}+\frac{1}{q}-\frac{1}{r}} \delta_{2}^{1-\frac{1}{r}} .
$$

Here $1 \leq p, q, r \leq \infty$, and $q^{\prime}, r^{\prime}$ are the exponents conjugate to $q, r$, respectively.

The notation $A \sim C$ means that the ratio $A / C$ is bounded above and below by quantities depending on $Z, \theta, A$, and the compact set $K$, but not on $\delta_{1}, \delta_{2}$. In the absence of weak comparability, the doubling property (i) fails in general for two-parameter Carnot-Carathéodory balls associated to $C^{\infty}$ vector fields satisfying the bracket condition; see [2].

For a sketch of the proof of Lemma 1, see $\$$ below.

Statement of results. Recall that $T$ is said to be of restricted weak type $(p, q)$ if for all Lebesgue measurable sets $E \subset X$ and $F \subset Y$,

$$
\left\langle T \chi_{E}, \chi_{F}\right\rangle \lesssim|E|^{1 / p}|F|^{1 / q^{\prime}},
$$

where $q^{\prime}$ denotes the exponent conjugate to $q$. In our setup,

$$
\left\langle T \chi_{E}, \chi_{F}\right\rangle \approx\left|\pi_{1}^{-1}(E) \cap \pi_{2}^{-1}(F)\right|,
$$

where $|\cdot|$ denotes Lebesgue measure on $Z$. We test the inequality (2) on the Carnot-Carathéodory balls $B\left(z, \delta_{1}, \delta_{2}\right)$ under the restriction that $\delta_{1} \sim{ }_{(\theta, A)} \delta_{2}$. Let $E=\pi_{1}\left(B\left(z, \delta_{1}, \delta_{2}\right)\right), F=\pi_{2}\left(B\left(z, \delta_{1}, \delta_{2}\right)\right)$. Using (3), Lemma 1 and restricting attention to the nontrivial case where $q>p$, the inequality (2) reads

$$
\left|B\left(z, \delta_{1}, \delta_{2}\right)\right| \gtrsim \delta_{1}^{c_{1}} \delta_{2}^{c_{2}}
$$

where

$$
c_{1}=\frac{p^{-1}}{p^{-1}-q^{-1}}, \quad c_{2}=\frac{1-q^{-1}}{p^{-1}-q^{-1}} .
$$

Define

$$
\mathcal{C}_{\theta, A}(T):=\left\{\left(c_{1}, c_{2}\right): \inf \left(\frac{\left|B\left(z, \delta_{1}, \delta_{2}\right)\right|}{\delta_{1}^{c_{1}} \delta_{2}^{c_{2}}}\right)>0\right\},
$$

where the infimum is taken over all $z \in Z$ and over all pairs $\delta_{1}, \delta_{2}$ that satisfy $\delta_{1} \sim_{(\theta, A)} \delta_{2}$. Define

$$
\mathcal{C}(T):=\bigcap_{0<\theta \leq 1} \bigcap_{A \geq 1} \mathcal{C}_{\theta, A}(T)
$$


According to (4), (2) cannot hold for $(p, q)$ if the corresponding $\left(c_{1}, c_{2}\right)$ does not belong to $\mathcal{C}(T)$. Tao and Wright [16] proved that 1 for all $\left(c_{1}, c_{2}\right)$ in the interior of $\mathcal{C}(T)$, (2) holds for the exponents $(p, q)$ defined by (4).

In this note we extend this result to mixed norms. We say that $T$ is of restricted weak mixed type $(p, q, r)$ if for all $E \subset X$ and $F \subset Y$,

$$
\left\langle T \chi_{E}, \chi_{F}\right\rangle \lesssim|E|^{1 / p}\left\|\chi_{F}\right\|_{q^{\prime}, r^{\prime}}
$$

By interpolation, the strong mixed type estimates can be obtained from these inequalities, except for exponents corresponding to boundary points of $\mathcal{C}(T)$.

The two-parameter Carnot-Carathéodory balls defined above also dictate the allowed exponent triples $(p, q, r)$ for mixed norm inequalities, under certain additional restrictions on the exponents $p, q, r$ :

Definition 3. Let $P_{\theta, A}(T)$ be the set of all exponents $(p, q, r)$ satisfying

(i) $1 \leq p \leq q \leq r \leq \infty$,

$$
\sup _{z, \delta_{1}, \delta_{2}} \frac{\left|B\left(z, \delta_{1}, \delta_{2}\right)\right|}{\left|\pi_{1}\left(B\left(z, \delta_{1}, \delta_{2}\right)\right)\right|^{1 / p}\left\|\chi_{\pi_{2}\left(B\left(z, \delta_{1}, \delta_{2}\right)\right)}\right\|_{q^{\prime}, r^{\prime}}}<\infty,
$$

where $q^{\prime}, r^{\prime}$ are the conjugates of $q, r$ respectively, and the supremum is taken over all $z \in Z$ and $\delta_{1} \sim_{\theta, A} \delta_{2}$.

Using Lemma 1 we can rewrite the second condition in the definition of $P_{\theta, A}(T)$ as in (4) with

$$
c_{1}=\frac{p^{-1}+q^{-1}-r^{-1}}{p^{-1}-r^{-1}}, \quad c_{2}=\frac{1-r^{-1}}{p^{-1}-r^{-1}}
$$

Define

$$
P(T):=\bigcap_{0<\theta \leq 1} \bigcap_{A \geq 1} P_{\theta, A}(T)=\left\{(p, q, r): r \geq q \geq p,\left(c_{1}, c_{2}\right) \in \mathcal{C}(T)\right\} .
$$

We assume always that $r>p$, since otherwise the desired inequality holds automatically so long as $\pi_{1}, \pi_{2}$ are submersions, as discussed above.

It is natural to conjecture that if $1 \leq p \leq q \leq r \leq \infty$, then $T$ is of restricted weak mixed type $(p, q, r)$ if and only if $(p, q, r) \in P(T)$; we prove this conjecture except at the endpoints. In an appendix we explain the presence of the additional restriction $p \leq q \leq r$.

Theorem 1. Let $T$ be a generalized Radon transform of the class described above, and let $1 \leq p \leq q \leq r \leq \infty$. If $(p, q, r)$ is in the interior of $P(T)$, then $T$ maps $L^{p}(X)$ to $L^{q} L^{r}(Y)$ boundedly. Moreover, if $(p, q, r) \notin P(T)$, then $T$ does not map $L^{p}(X)$ to $L^{q} L^{r}(Y)$ boundedly.

\section{A BRIEF ACCOUnt of [16]}

Given $E \subset X$ and $F \subset Y$, let

$$
\alpha_{1}=\frac{\left\langle T \chi_{E}, \chi_{F}\right\rangle}{|E|}, \quad \alpha_{2}=\frac{\left\langle T \chi_{E}, \chi_{F}\right\rangle}{|F|} .
$$

\footnotetext{
${ }^{1}$ Tao and Wright defined the set $\mathcal{C}(T)$ differently. An analysis of the two-parameter balls along the lines of 11] establishes the equivalence of these two definitions.
} 
(2) follows from an estimate of the form

$$
|\Omega|:=\left|\pi_{1}^{-1}(E) \cap \pi_{2}^{-1}(F)\right| \approx\left\langle T \chi_{E}, \chi_{F}\right\rangle \gtrsim \alpha_{1}^{c_{1}} \alpha_{2}^{c_{2}},
$$

where $c_{1}, c_{2}$ are as in (5). Moreover, a loss of an arbitrarily small power of $\alpha_{1} \alpha_{2}$ is of no consequence since $\left(c_{1}, c_{2}\right)$ belongs to the interior of $\mathcal{C}(T)$. Throughout the remainder of the discussion we will denote

$$
\Omega:=\pi_{1}^{-1}(E) \cap \pi_{2}^{-1}(F) .
$$

Let $\varepsilon>0$ be a small exponent to be specified near the end of the proof, and let $C_{\varepsilon}$ be a large constant. All constants in the ensuing discussion depend on $X, Y, Z, c_{1}, c_{2}$ and the quantities given as subscripts.

Definition 4. Let $S \subset[-1,1]$ be a measurable set of positive Lebesgue measure. We say that $S$ is central with width $w>0$ if

(i) $S \subset\left[-C_{\varepsilon} w, C_{\varepsilon} w\right]$ and

(ii) $|I \cap S| \leq C_{\varepsilon}(|I| / w)^{\varepsilon}|S|$ for all intervals $I$.

Let $\Omega$ be a Lebesgue measurable subset of $Z$, having positive measure. We will assume throughout the discussion that $\pi_{j}(\Omega)$ has positive measure, for both $j=1,2$. Define

$$
\begin{gathered}
\alpha_{j}=\frac{|\Omega|}{\left|\pi_{j}(\Omega)\right|} \text { for } j=1,2, \\
\alpha:=\min \left(\alpha_{1}, \alpha_{2}\right) .
\end{gathered}
$$

Fix any $\rho>0 ; \rho$ will eventually be chosen to be arbitrarily small at the conclusion of the proof.

Definition 5. Let $\Omega \subset Z$ and let $\alpha_{1}, \alpha_{2}$ be as defined in (10). Let $j$ be an integer. A $j$-subsheaf $\Omega^{\prime}$ of $\Omega$, of width $w_{j}$, is a subset of $\Omega$ of measure $\geq C_{\rho}^{-1} \alpha^{\rho}|\Omega|$ such that for all $x \in \Omega^{\prime}$, the set

$$
\left\{|t| \ll 1: e^{t V_{j}}(x) \in \Omega^{\prime}\right\}
$$

is a central set of width $w_{j}$ and measure $\geq C_{N, \varepsilon} \alpha^{C_{N} \varepsilon+C / N} \alpha_{j}$. Here $V_{j}=V_{1}$ if $j$ is odd, and $V_{j}=V_{2}$ if $j$ is even.

In [16] it is proved (see Corollary 8.3) that for any $\rho \in(0,1]$ there exists $C_{\rho}<\infty$ such that for any measurable set $\Omega \subset Z$ there exists a nested sequence of subsets of $\Omega$

$$
\Omega_{0} \subset \Omega_{1} \subset \ldots \subset \Omega_{d+1} \subset \Omega
$$

such that: For each $j, \Omega_{j}$ is a $j$-sheaf of $\Omega$ with width $w_{j}$,

$$
C_{\rho}^{-1} \alpha^{\rho} \delta_{j} \leq w_{j} \leq \delta_{j},
$$

where $\delta_{j}$ is a 2 -periodic sequence (that is, $\delta_{j+2}=\delta_{j}$ for all $0 \leq j \leq d-1$ ) with the properties

$$
C_{\rho}^{-1} \alpha^{\rho} \alpha_{j} \leq \delta_{j} \leq 1,
$$

and

$$
\delta_{1} \leq C_{\rho} \delta_{2}^{\rho}, \quad \delta_{2} \leq C_{\rho} \delta_{1}^{\rho} .
$$

In particular, $\delta_{1}, \delta_{2}$ are weakly comparable, even though $\alpha_{1}, \alpha_{2}$ need not be; this ultimately explains why only balls with weakly comparable radii $\delta_{1}, \delta_{2}$ need be taken into account in the hypothesis of our theorem. 
Using this construction, it is proved [16] that there exists some ball $B\left(z, \delta_{1}, \delta_{2}\right)$ such that

$$
\left|B\left(z, \delta_{1}, \delta_{2}\right) \cap \Omega\right| \geq c \alpha^{\varrho}\left(\frac{\alpha_{1}}{\delta_{1}}\right)^{\lfloor(d+2) / 2\rfloor}\left(\frac{\alpha_{2}}{\delta_{2}}\right)^{\lfloor(d+1) / 2\rfloor}\left|B\left(z, \delta_{1}, \delta_{2}\right)\right|,
$$

where $\varrho>0$ can be made arbitrarily small by choosing $\rho$ sufficiently small, and where $c>0$ depends on $\varrho$ but not on $\Omega, \alpha_{j}, \delta_{j}$. This implies that for arbitrarily small $\varrho>0$,

$$
|\Omega| \gtrsim C_{\varrho} \alpha^{\varrho} \delta_{1}^{c_{1}} \delta_{2}^{c_{2}}\left(\frac{\alpha_{1}}{\delta_{1}}\right)^{\lfloor(d+2) / 2\rfloor}\left(\frac{\alpha_{2}}{\delta_{2}}\right)^{\lfloor(d+1) / 2\rfloor},
$$

for all $\left(c_{1}, c_{2}\right)$ in the interior of $\mathcal{C}(T)$. This finishes the proof in the nonmixednorm case since $2 c_{1}, c_{2} \geq d \geq\lfloor(d+2) / 2\rfloor,\lfloor(d+1) / 2\rfloor$. The roles of $X$ and $Y$ are interchangeable. Therefore there is the alternative bound

$$
|\Omega| \gtrsim C_{\varrho} \alpha^{\varrho} \delta_{1}^{c_{1}} \delta_{2}^{c_{2}}\left(\frac{\alpha_{1}}{\delta_{1}}\right)^{\lfloor(d+1) / 2\rfloor}\left(\frac{\alpha_{2}}{\delta_{2}}\right)^{\lfloor(d+2) / 2\rfloor},
$$

for all $\left(c_{1}, c_{2}\right)$ in the interior of $\mathcal{C}(T)$.

\section{Proof of Theorem 1}

The second conclusion of the theorem is immediate. If $(p, q, r) \notin P(T)$, then $(p, q, r) \notin P_{\theta, A}(T)$ for some $\theta, A$. Therefore $T$ cannot be of mixed type $(p, q, r)$.

For the first conclusion, it suffices to prove that for all $(p, q, r)$ in the interior of $P(T)$ and for all $\eta>0, \beta>0$, and $F \subset Y$,

$$
\left\langle T \chi_{E}, \chi_{F}\right\rangle \leq C_{p, q, r, \eta} \beta^{-\eta}|E|^{1 / p}\left\|\chi_{F}\right\|_{q^{\prime}, r^{\prime}},
$$

where $E:=\left\{x \in X: \beta<T^{*} \chi_{F}(x) \leq 2 \beta\right\}$.

Let $\Omega:=\pi_{1}^{-1}(E) \cap \pi_{2}^{-1}(F)$. Note that

$$
|\Omega| \approx\left\langle T \chi_{E}, \chi_{F}\right\rangle=\left\langle\chi_{E}, T^{*} \chi_{F}\right\rangle \approx \beta|E| .
$$

For each $x \in E$, let $\mathcal{F}(x):=\left\{t \in \mathbf{R}: \gamma_{x}^{*}(t) \in F\right\} \subset \Pi(F) \subset[-1,1]$. Note that $|\mathcal{F}(x)| \approx \beta$.

Let $\eta>0$ be a small constant to be specified at the end of the proof. Fix a small constant $c_{\eta}>0$. Let $I(x) \subset[-1,1]$ be a dyadic interval of minimal length so that

$$
|I(x) \cap \mathcal{F}(x)| \geq c_{\eta}|I(x)|^{\eta}|\mathcal{F}(x)| ;
$$

we choose $c_{\eta}$ to guarantee that either $I=[-1,0]$ or $I=[0,1]$ satisfies (17). No interval of length $<\left(c_{\eta}|\mathcal{F}(x)|\right)^{1 /(1-\eta)}$ can satisfy (17), so there must exist at least one dyadic interval of minimal length among all those satisfying the inequality.

This implies that for any dyadic subinterval $J \subset I(x)$,

$$
|J \cap \mathcal{F}(x)| \leq \frac{|J|^{\eta}}{|I|^{\eta}}|I(x) \cap \mathcal{F}(x)| .
$$

Let

$$
E_{m, k}=\left\{x \in E:|I(x)| \approx 2^{m} \beta,|I(x) \cap \mathcal{F}(x)| \approx 2^{k}\right\} .
$$

Note that $\beta^{\eta /(1-\eta)} \lesssim 2^{m} \lesssim \beta^{-1}$ and $\beta^{1 /(1-\eta)} \lesssim 2^{k} \lesssim \beta$. [16].

${ }^{2}$ The bounds $c_{1}, c_{2} \geq d$ are a simple consequence of an equivalent definition of $\mathcal{C}(T)$ given in 
By the pigeonhole principle there exists a pair $m, k$ such that $\tilde{E}=E_{m, k}$ satisfies

$$
\left\langle T \chi_{E}, \chi_{F}\right\rangle \leq C_{\eta} \beta^{-\eta}\left\langle T \chi_{\tilde{E}}, \chi_{F}\right\rangle \text {. }
$$

Choose one such pair, with which we work exclusively henceforth.

Partition $\mathbf{R}$ into intervals $I_{n}$, each of length $C 2^{m} \beta$ where $C$ is a sufficiently large fixed constant. Set

$$
\begin{gathered}
E_{n}=\left\{x \in \tilde{E}: I(x) \cap I_{n} \neq \emptyset\right\}, \\
F_{n}=F \cap \Pi^{-1}\left(I_{n-1} \cup I_{n} \cup I_{n+1}\right) .
\end{gathered}
$$

Note that

$$
\left\langle T \chi_{\tilde{E}}, \chi_{F}\right\rangle \lesssim \sum_{n}\left\langle T \chi_{E_{n}}, \chi_{F_{n}}\right\rangle
$$

Let $\Omega^{n}:=\pi_{1}^{-1}\left(E_{n}\right) \cap \pi_{2}^{-1}\left(F_{n}\right)$. Then

$$
2^{k}\left|E_{n}\right| \lesssim\left|\Omega^{n}\right| \leq \beta\left|E_{n}\right|
$$

by Fubini's theorem. On the one hand, $\left|\pi_{1}^{-1}(x)\right| \leq \beta$ for all $x \in E$. On the other hand, $2^{k} \sim|I(x) \cap \mathcal{F}(x)|$ and for $x \in E_{n}, I(x) \cap \mathcal{F}(x) \subset\left(I_{n-1} \cup I_{n} \cup I_{n+1}\right) \cap \mathcal{F}(x) \subset$ $\pi_{2}^{-1}\left(F_{n}\right)$. Using (18) with $I=I_{n-1} \cup I_{n} \cup I_{n+1}$, for each $x \in E_{n}$ and any dyadic interval $J$, we obtain

$$
|J \cap \mathcal{F}(x)| \lesssim|J|^{\eta}\left(2^{m} \beta\right)^{-\eta} 2^{k} .
$$

Let $\alpha_{n, i}=\left|\Omega^{n}\right| /\left|\pi_{i}\left(\Omega^{n}\right)\right|, i=1,2$. Then

$$
2^{k} \lesssim \alpha_{n, 1} \lesssim \beta,
$$

by (21). Let $\alpha_{n}=\min \left(\alpha_{n, 1}, \alpha_{n, 2}\right)$.

The following lemma follows from a simple application of Hölder's inequality; see [4].

Lemma 2. Let $F \subset Y$. For $r \geq q$,

$$
\left\|\chi_{F}\right\|_{q^{\prime} r^{\prime}} \geq|F|^{1 / r^{\prime}}|\Pi(F)|^{1 / q^{\prime}-1 / r^{\prime}} .
$$

We aim to prove that $\left\langle T \chi_{E_{n}}, \chi_{F_{n}}\right\rangle \lesssim\left|E_{n}\right|^{1 / p}\left\|\chi_{F_{n}}\right\|_{q^{\prime}, r^{\prime}}$. Via the preceding lemma, this would follow from

$$
\left|\Omega^{n}\right| \approx\left\langle T \chi_{E_{n}}, \chi_{F_{n}}\right\rangle \gtrsim \alpha_{n, 1}^{\gamma_{1}} \alpha_{n, 2}^{\gamma_{2}}\left|\Pi\left(F_{n}\right)\right|^{\gamma_{3}},
$$

where

$$
\gamma_{1}=\frac{p^{-1}}{p^{-1}-r^{-1}}, \quad \gamma_{2}=\frac{1-r^{-1}}{p^{-1}-r^{-1}}, \quad \gamma_{3}=\frac{q^{-1}-r^{-1}}{p^{-1}-r^{-1}} .
$$

Whereas the exponent $\frac{1}{q^{\prime}}-\frac{1}{r^{\prime}}$ in Lemma 2 was nonpositive, here all the exponents $\gamma_{j}$ are nonnegative.

The construction of Tao and Wright [16] produces a nested sequence of subsets $\Omega_{0}^{n} \subset \Omega_{1}^{n} \subset \ldots \subset \Omega_{d+1}^{n} \subset \Omega^{n}$ satisfying: For each $j, \Omega_{j}^{n}$ is a $j$-sheaf of $\Omega^{n}$ with width $w_{j}$,

$$
C_{\rho}^{-1} \alpha_{n}^{\rho} \delta_{j} \leq w_{j} \leq C_{\rho} \delta_{j},
$$

where $\delta_{j}$ is a 2-periodic sequence with the property

$$
C_{\rho}^{-1} \alpha_{n}^{\rho} \alpha_{n, j} \leq \delta_{j} \leq 1,
$$

and $\left|\Omega_{j}^{n}\right| \geq c_{\rho} \alpha_{n}^{\rho}\left|\Omega^{n}\right|$. Moreover, $\delta_{1}, \delta_{2}$ are weakly comparable. 
Now, we prove that the construction above guarantees a lower bound for $\delta_{1}$. Indeed, let $j$ be odd. Using (22), we have

$$
\left|\Omega_{j}^{n}\right| \leq\left|E_{n}\right| \sup _{J, x}\left|J \cap F_{n}(x)\right| \leq\left|E_{n}\right| w_{j}^{\eta}\left(2^{m} \beta\right)^{-\eta} 2^{k},
$$

where the supremum is taken over all intervals $J$ of length $w_{j}$ and all points $x \in E_{n}$. Using (27), (21) and the fact that $\Omega_{j}^{n}$ is a $j$-sheaf of $\Omega^{n}$, we get

$$
c_{\rho} \alpha_{n}^{\rho} 2^{k}\left|E_{n}\right| \lesssim C_{\rho} \alpha_{n}^{\rho}\left|\Omega^{n}\right| \leq\left|\Omega_{j}^{n}\right| \leq\left|E_{n}\right| w_{j}^{\eta}\left(2^{m} \beta\right)^{-\eta} 2^{k} .
$$

This implies that for odd $j$,

$$
\delta_{1} \gtrsim w_{j} \gtrsim 2^{m} \beta C_{\rho}^{1 / \eta} \alpha_{n}^{\rho / \eta} \gtrsim\left|\Pi\left(F_{n}\right)\right| C_{\rho}^{1 / \eta} \alpha_{n}^{\rho / \eta} .
$$

Using the fundamental information (14), we obtain

$$
\left|\Omega^{n}\right| \gtrsim \alpha_{n}^{\varrho} \alpha_{n}^{C \rho / \eta} \delta_{1}^{c_{1}} \delta_{2}^{c_{2}}\left(\frac{\alpha_{n, 1}}{\delta_{1}}\right)^{\lfloor(d+1) / 2\rfloor}\left(\frac{\alpha_{n, 2}}{\delta_{2}}\right)^{\lfloor(d+2) / 2\rfloor},
$$

for any $\left(c_{1}, c_{2}\right)$ belonging to the interior of $\mathcal{C}(T)$. Note that for $d \geq 2$ and for such $\left(c_{1}, c_{2}\right)$, we have $0 \leq \gamma_{3}<1 \leq c_{1}-\lfloor(d+1) / 2\rfloor$ and $c_{2}>\lfloor(d+2) / 2\rfloor$. Using this, (26) and (29), we obtain

$$
\left|\Omega^{n}\right| \gtrsim \alpha_{n}^{\varrho} \alpha_{n}^{C \rho / \eta} \delta_{1}^{\gamma_{3}} \alpha_{n, 1}^{c_{1}-\gamma_{3}} \alpha_{n, 2}^{c_{2}} \gtrsim \alpha_{n}^{\varrho} \alpha_{n}^{C \rho / \eta}\left|\Pi\left(F_{n}\right)\right|^{\gamma_{3}} \alpha_{n, 1}^{c_{1}-\gamma_{3}} \alpha_{n, 2}^{c_{2}},
$$

for all $\left(c_{1}, c_{2}\right)$ in the interior of $\mathcal{C}(T)$.

Given exponents $(p, q, r) \in P(T)$, define $\gamma_{1}, \gamma_{2}, \gamma_{3}$ by (25). Choose $\rho>0$, depending on $\eta$, and $\left(c_{1}, c_{2}\right)$ in the interior of $\mathcal{C}(T)$, so that

$$
\frac{C \rho}{\eta}+c_{1}-\gamma_{3}+\varrho \leq \gamma_{1}, \quad \frac{C \rho}{\eta}+c_{2}+\varrho \leq \gamma_{2} .
$$

Therefore, since $\gamma_{3} \geq 0$ (which is a consequence of the assumptions that $r>p$ and $r \geq q)$,

$$
\left|\Omega^{n}\right| \gtrsim \alpha_{n, 1}^{\gamma_{1}} \alpha_{n, 2}^{\gamma_{2}}\left|\Pi\left(F_{n}\right)\right|^{\gamma_{3}},
$$

and hence

$$
\left\langle T \chi_{E_{n}}, \chi_{F_{n}}\right\rangle \lesssim\left|E_{n}\right|^{1 / p}\left\|\chi_{F_{n}}\right\|_{q^{\prime}, r^{\prime}} .
$$

Using this and then Hölder's inequality in (20) (here we use the assumption $q \geq p$ ), we have

$$
\begin{aligned}
\left\langle T \chi_{E}, \chi_{F}\right\rangle & \lesssim \beta^{-\eta} \sum_{n}\left\langle T \chi_{E_{n}}, \chi_{F_{n}}\right\rangle \\
& \lesssim \beta^{-\eta} \sum_{n}\left|E_{n}\right|^{1 / p}\left\|\chi_{F_{n}}\right\|_{q^{\prime}, r^{\prime}} \\
& \lesssim \beta^{-\eta}\left[\sum_{n}\left|E_{n}\right|\right]^{1 / p}\left[\sum_{n}\left\|\chi_{F_{n}}\right\|_{q^{\prime}, r^{\prime}}^{q^{\prime}}\right]^{1 / q^{\prime}} \\
& \leq \beta^{-\eta}|E|^{1 / p}\left\|\chi_{F}\right\|_{q^{\prime}, r^{\prime}} .
\end{aligned}
$$

In the last inequality we have used the bounded overlap property of the collections $\left\{E_{n}\right\}$ and $\left\{F_{n}\right\}$. Since $\eta>0$ can be chosen to be arbitrarily small, this finishes the proof of Theorem 1 


\section{Proof of Lemma 1}

We refer the reader to [2] for the proofs of (i) and (ii).

Let $B$ be the ball $B\left(z_{0}, \delta_{1}, \delta_{2}\right)$. Note that $\left\{e^{s V_{1}} z_{0}:|s|<\delta_{1}\right\} \subset B$ and by (1), $\left|\Pi \pi_{2}\left(\left\{e^{s V_{1}} z_{0}:|s|<\delta_{1}\right\}\right)\right| \approx \delta_{1}$. Now, we prove that $\left|\Pi \pi_{2}(B)\right| \lesssim \delta_{1}$. Let $z \in B$ and let $\varphi$ be a curve connecting $z_{0}$ to $z$ as in Definition 1. Let $\psi=\Pi \circ \pi_{2} \circ \varphi$. Since $V_{2}$ is contained in the kernel of $D \pi_{2},\left|a_{1}(t)\right|<\delta_{1}$ and $\left|V_{1}\right| \lesssim 1$, we have

$$
\left|\psi^{\prime}(t)\right| \lesssim \delta_{1}
$$

Therefore $\left|\Pi \pi_{2}(B)\right| \lesssim \delta_{1}$. This proves (iii).

We have actually shown that the 1-dimensional measure of $B\left(z_{0}, 2 \delta_{1}, 2 \delta_{2}\right) \cap$ $\pi_{1}^{-1}(x)$ is $\approx \delta_{1}$ for every $x \in \pi_{1}(B)$. This also proves (ii) for $j=1$. In fact it implies that if $A$ is a subset of a $k$-dimensional smooth submanifold of $X$, then $\delta_{1}$ times the $k$-dimensional measure of $A$ is comparable to the $(k+1)$-dimensional measure of $\pi_{1}^{-1}(A) \cap B$. The corresponding statement holds for $j=2$, as well.

To prove (iv), define $f\left(t, \delta_{1}, \delta_{2}\right)=\left|\pi_{2}^{-1} \Pi^{-1}(t) \cap B\left(z_{0}, \delta_{1}, \delta_{2}\right)\right|$, where $|\cdot|$ signifies the $d$-dimensional measure of a subset of $Z$. To simplify notation we suppose that $\Pi\left(\pi_{2}\left(z_{0}\right)\right)=0$; this can be achieved by a change of coordinates. Note that if $A \subset B\left(z_{0}, \delta_{1}, \delta_{2}\right)$, then for $s<\delta_{1}, e^{s V_{1}} A \subset B\left(z_{0}, 2 \delta_{1}, 2 \delta_{2}\right)$. Choose $t_{0} \in\left[-\delta_{1}, \delta_{1}\right]$ so that $f\left(t_{0}, \delta_{1}, \delta_{2}\right)=\max _{|t| \leq \delta_{1}} f\left(t, \delta_{1}, \delta_{2}\right)$. Then

$$
\left|B\left(z_{0}, 2 \delta_{1}, 2 \delta_{2}\right)\right| \gtrsim \int_{|s| \leq \delta_{1}}\left|e^{s V_{1}} f\left(t_{0}\right)\right| d s \gtrsim \delta_{1} f\left(t_{0}\right) .
$$

Now $\left|B\left(z_{0}, \delta_{1}, \delta_{2}\right)\right| \gtrsim\left|B\left(z_{0}, 2 \delta_{1}, 2 \delta_{2}\right)\right|$; the proof given by Nagel, Stein, and Wainger 11 of the volume doubling property carries over to two-parameter balls with $(\theta, A)-$ weakly comparable radii, with a bound depending on $\theta$ and on $A$ but not on $\delta_{1}, \delta_{2}, z_{0}$. We conclude that $f(t) \lesssim\left|B\left(z_{0}, \delta_{1}, \delta_{2}\right)\right| / \delta_{1}$ whenever $|t| \leq \delta_{1}$.

Similar reasoning shows that if $|t|,\left|t^{\prime}\right| \leq \delta_{1}$, then $f\left(t^{\prime}, \delta_{1}, \delta_{2}\right) \leq C f\left(t, 3 \delta_{1}, 3 \delta_{2}\right)$. Since $\Pi\left(\pi_{2}\left(B\left(z_{0}, \delta_{1}, \delta_{2}\right)\right) \subset\left[-\delta_{1}, \delta_{1}\right]\right.$, there exists some $t^{\prime} \in\left[-\delta_{1}, \delta_{1}\right]$ for which $f\left(t^{\prime}, \delta_{1}, \delta_{2}\right) \gtrsim\left|B\left(z_{0}, \delta_{1}, \delta_{2}\right)\right| / \delta_{1}$. By combining these two observations, we conclude the reverse inequality $f\left(t, 3 \delta_{1}, 3 \delta_{2}\right) \gtrsim\left|B\left(z_{0}, \delta_{1}, \delta_{2}\right)\right| / \delta_{1}$ whenever $|t| \leq \delta_{1}$.

The lower bound for $\left\|\chi_{\pi_{2}(B)}\right\|_{q^{\prime}, r^{\prime}}$ in (iv) follows from this together with the observation that $f(t) \approx\left|\pi_{2}(f(t))\right| \delta_{2}$. The upper bound follows in the same way from the upper bound $f(t) \lesssim\left|B\left(z_{0}, \delta_{1}, \delta_{2}\right)\right| / \delta_{1}$.

Finally, conclusion (v) follows from (ii) and (iv).

\section{ON THE HYPOTHESIS $p \leq q \leq r$}

One reason why the restriction $r \geq q \geq p$ in Theorem 1 is natural is as follows.

Proposition 3. Suppose that $\left|B\left(z, \delta_{1}, \delta_{2}\right)\right|$ is comparable to $\left|B\left(z^{\prime}, \delta_{1}, \delta_{2}\right)\right|$, uniformly for all $z, z^{\prime}$ and all weakly comparable $\delta_{1}, \delta_{2}$. Then all valid mixed norm inequalities for $T$ are implied by the conjectured inequalities. That is:

(i) If $T$ is of restricted weak mixed type $(p, q, r)$ with $r>p>q$, then $(p, p, r) \in$ $P(T)$.

(ii) If $T$ is of restricted weak mixed type $(p, q, r)$ with $q>r>p$, then $(p, q, r)$ is an interpolant between $(1, \infty, 1)$ and some $\left(p_{1}, q_{1}, r_{1}\right) \in P(T)$.

In case (i), the restricted weak mixed type bound for $(p, p, r)$ implies that for $(p, q, r)$ by Hölder's inequality, since we are working in a bounded region and $q<p$. In case (ii), the conclusion is that $\left(p^{-1}, q^{-1}, r^{-1}\right)$ belongs to the line segment with 
endpoints $(1,0,1)$ and $\left(p_{1}^{-1}, q_{1}^{-1}, r_{1}^{-1}\right)$. Since any generalized Radon transform $T$ is of strong type $(1, \infty, 1)$, the restricted weak mixed type $(p, q, r)$ inequality follows from the $\left(p_{1}, q_{1}, r_{1}\right)$ inequality by interpolation.

Proof. (i) If $(p, p, r) \notin P(T)$, then there exist $\theta, A$ and a sequence of balls $B_{n}\left(z_{n}, \delta_{n, 1}, \delta_{n, 2}\right)$ with $\delta_{n, 1} \sim_{(\theta, A)} \delta_{n, 2}$ satisfying

$$
\left|B_{n}\right|^{\frac{1}{r}-\frac{1}{p}} \delta_{n, 1}^{\frac{2}{p}-\frac{1}{r}} \delta_{n, 2}^{1-\frac{1}{r}} \rightarrow \infty
$$

as $n \rightarrow \infty$. Choose $N_{n} \approx \delta_{n, 1}^{-1}$ balls of size comparable to $B_{n}$ with disjoint projections under $\Pi \circ \pi_{2}$; this is possible by Lemma 1. Let $U_{n}$ be the union of these balls. Note that $\left|\pi_{1}\left(U_{n}\right)\right| \lesssim N_{n}\left|\pi_{1}\left(B_{n}\right)\right|$.

$$
\begin{aligned}
\frac{\left|U_{n}\right|}{\left|\pi_{1}\left(U_{n}\right)\right|^{1 / p}\left\|\chi_{\pi_{2}\left(U_{n}\right)}\right\|_{q^{\prime}, r^{\prime}}} & \gtrsim \frac{N_{n}\left|B_{n}\right|}{\left[N_{n} \frac{\left|B_{n}\right|}{\delta_{n, 1}}\right]^{1 / p}\left[\frac{\left|B_{n}\right|}{\delta_{n, 1} \delta_{n, 2}}\right]^{1 / r^{\prime}} \delta_{n, 1}^{1 / q^{\prime}} N_{n}^{1 / q^{\prime}}} \\
& \approx\left(\left|B_{n}\right|^{\frac{1}{r}-\frac{1}{p}} \delta_{n, 1}^{\frac{2}{p}-\frac{1}{r}} \delta_{n, 2}^{1-\frac{1}{r}}\right)\left(N_{n} \delta_{n, 1}\right)^{1 / q-1 / p} \\
& \approx\left|B_{n}\right|^{\frac{1}{r}-\frac{1}{p}} \delta_{n, 1}^{\frac{2}{p}-\frac{1}{r}} \delta_{n, 2}^{1-\frac{1}{r}} \rightarrow \infty,
\end{aligned}
$$

as $n \rightarrow \infty$. Thus, $T$ cannot be of restricted weak mixed type $(p, q, r)$.

(ii) For $s \in[0,1]$ define $p_{1}, q_{1}, r_{1}$ by

$$
\frac{1}{p}=1-s+\frac{s}{p_{1}}, \quad \frac{1}{q}=\frac{s}{q_{1}}, \quad \frac{1}{r}=1-s+\frac{s}{r_{1}} .
$$

A bit of algebra shows that $r_{1} \geq q_{1} \geq p_{1}$ if and only if $\frac{1}{q}+\frac{1}{p^{\prime}} \leq s \leq \frac{1}{q}+\frac{1}{r^{\prime}}$, and moreover that $0<\frac{1}{q}+\frac{1}{p^{\prime}} \leq \frac{1}{q}+\frac{1}{r^{\prime}}<1$ under our assumption that $q>r>p$. Thus it is possible to choose $s \in(0,1)$ so that $r_{1} \geq q_{1} \geq p_{1}$. Fix such a parameter $s$.

If $\left(p_{1}, q_{1}, r_{1}\right) \in P(T)$, then we have the conclusion of case (ii). Otherwise there exist $\theta, A$ and a sequence of balls $B_{n}=B_{n}\left(z_{n}, \delta_{n, 1}, \delta_{n, 2}\right)$ with $\delta_{n, 1} \sim_{(\theta, A)} \delta_{n, 2}$ such that

$$
\left|B_{n}\right|^{\frac{1}{r_{1}}-\frac{1}{p_{1}}} \delta_{n, 1}^{\frac{1}{p_{1}}+\frac{1}{q_{1}}-\frac{1}{r_{1}}} \delta_{n, 2}^{1-\frac{1}{r_{1}}} \rightarrow \infty
$$

Note that for these balls,

$$
\begin{aligned}
\frac{\left|B_{n}\right|}{\left|\pi_{1}\left(B_{n}\right)\right|^{1 / p}\left\|\chi_{\pi_{2}\left(B_{n}\right)}\right\|_{q^{\prime}, r^{\prime}}} & \approx\left|B_{n}\right|^{\frac{1}{r}-\frac{1}{p}} \delta_{n, 1}^{\frac{1}{p}+\frac{1}{q}-\frac{1}{r}} \delta_{n, 2}^{1-\frac{1}{r}} \\
& =\left(\left|B_{n}\right|^{\frac{1}{r_{1}}-\frac{1}{p_{1}}} \delta_{n, 1}^{\frac{1}{p_{1}}+\frac{1}{q_{1}}-\frac{1}{r_{1}}} \delta_{n, 2}^{1-\frac{1}{r_{1}}}\right)^{s} \rightarrow \infty .
\end{aligned}
$$

Thus $T$ cannot be of restricted weak mixed type $(p, q, r)$.

Case (ii) is valid for all operators $T$, without the hypothesis that balls of equal bi-radii have uniformly comparable measures.

\section{REFERENCES}

1. M. Christ, Convolution, curvature, and combinatorics. A case study, Internat. Math. Research Notices 19 (1998), 1033-1048. Edinburgh, April 1-2, 2002. MR1654767(2000a:42026)

2. L Lebesgue space bounds for one-dimensional generalized Radon transforms, preprint.

3. _ Quasi-extremals for a Radon-like transform, preprint.

4. M. Christ and M. B. Erdoğan, Mixed norm estimates for a restricted X-ray transform, J. d'Analyse 87 (2002), 187-198. MR.1945281(2003k:42030) 
5. M. Christ, A. Nagel, E. M. Stein, and S. Wainger, Singular Radon transforms: Analysis and geometry, Ann. of Math. (2) 150 (1999), 489-577. MR1726701 (2000j:42023)

6. M. B. Erdoğan, Mixed norm estimates for a restricted X-ray transform in $\mathbf{R}^{4}$ and $\mathbf{R}^{5}$, Internat. Math. Research Notices 11 (2001), 575-600. MR.1836731 (2002i:44003)

7. A. Greenleaf and A. Seeger, Fourier integral operators with fold singularities, J. Reine Angew. Math. 455 (1994), 35-56. MR.1293873 (95h:58130)

8. , Fourier integral operators with cusp singularities, Amer. Jour. Math. 120 (1998), 1077-1119. MR1646055 (99g:58120)

9. - On X-ray transforms for rigid line complexes and integrals over curves in $\mathbf{R}^{4}$, Proc. Amer. Math. Soc. 127 (1999), 3533-3545. MR1670367 (2001a:44002)

10. A. Greenleaf, A. Seeger, and S. Wainger, Estimates for generalized Radon transforms in three and four dimensions, Analysis, geometry, number theory: The mathematics of Leon Ehrenpreis (Philadelphia, PA, 1998), 243-254, Contemp. Math., 251, Amer. Math. Soc., Providence, RI, 2000. MR1771272 (2001j:58047)

11. A. Nagel, E. Stein, and S. Wainger, Balls and metrics defined by vector fields I. Basic properties, Acta. Math. 155 (1985), 103-147. MR793239 (86k:46049)

12. D. H. Phong and E. M. Stein, Models of degenerate Fourier integral operators and Radon transforms, Ann. of Math. (2) 140 (1994), 703-722. MR.1307901 (96c:35206)

13. - The Newton polyhedron and oscillatory integral operators, Acta Math. 179 (1997), 105-152. MR:1484770 (98j:42009)

14. - Damped oscillatory integral operators with analytic phases, Adv. Math. 134 (1998), 146-177. MR1612395 (2000b:42009)

15. A. Seeger, Radon transforms and finite type conditions, J. Amer. Math. Soc. 11 (1998), 869897. MR1623430 (99f:58202)

16. T. Tao and J. Wright, $L^{p}$ improving bounds for averages along curves, J. Amer. Math. Soc. 16 (2003), 605-638. MR1969206 (2004j:42005)

17. T. Wolff, A sharp bilinear cone restriction estimate, Ann. of Math. (2) 153 (2001), 661-698. MR.1836285 (2002j:42019)

Department of Mathematics, University of California, Berkeley, California 947203840

E-mail address: mchrist@math.berkeley.edu

Department of Mathematics, University of Illinois, Urbana, Illinois 61801

E-mail address: berdogan@math.uiuc.edu 